\title{
Percepción de la calidad de la educación continua en un hospital de alta especialidad
}

\section{Perception of the quality of continuing education in a high specialty hospital}

\author{
Daniel E. Sánchez-Hinojosa ${ }^{1}$, Patricia Rivera-Vázquez ${ }^{2 *}$ y Sandra Rosales-Gracia ${ }^{3}$ \\ ${ }^{1}$ División de Enseñanza e Investigación, Hospital Regional de Alta Especialidad, Ciudad Victoria; ${ }^{2}$ Coordinación de Investigación en Enfermería, Hospital Regional de \\ Alta Especialidad, Ciudad Victoria; ${ }^{3}$ Departamento de Investigación, Área de Ciencias de la Salud, Universidad del Noreste, Tampico. Tamaulipas, México
}

\begin{abstract}
Research objective: To determine the association between age and professional profile of attendees to continuing education courses with the perception of their quality in a third-level hospital. Methods: We conducted an observational, descriptive and cross-sectional study, including 4,273 attendees through evaluation cards of the course (Hospital Regional de Alta Especialidad de Ciudad Victoria, 2013). Data were analyzed using descriptive and inferential statistics with SPSS ${ }^{\circledR}$ program v.21. Results: Female users $76.7 \%(3,277)$, average age 24.37 years, nurses $25.4 \%(1,086)$, doctors $8.7 \%$ (370), social workers $6.0 \%(258)$, and other degrees $5.2 \%(224)$. Internal users $46.1 \%(1,969)$, external $41.0 \%(1,751)$, and not specifying origin $12.9 \%$ (553). With a good perception of the quality of the courses for $77.5 \%(3,310)$ of the users, the evaluation of the teachers was perceived as good by $100 \%(4,273)$ of the users. Conclusion: No association was found between age and perceived quality $\left(\chi^{2}: 164.05 ; p=0.06\right)$, but it was between professional profile and perceived quality $\left(\chi^{2}: 72.78 ; p=0.00\right)$.
\end{abstract}

Key words: Perception. Quality. Continuing education.

\section{Resumen}

Objetivo de la investigación: Determinar la asociación de la edad y perfil profesional de los asistentes con la percepción de la calidad de los cursos de educación continua en un hospital de tercer nivel de atención. Métodos: Se realizó un estudio observacional, descriptivo y transversal, con 4,273 usuarios por medio de cédulas de evaluación del evento educativo (Hospital Regional de Alta Especialidad de Ciudad Victoria, 2013). Los datos se analizaron utilizando estadística descriptiva e inferencial con el programa SPSS ${ }^{\circledR}$ v.21. Resultados: Usuarias del sexo femenino, $76.7 \%$ (3,277); edad promedio, 24.37 años; enfermeras, 25.4\% (1,086); médicos, 8.7\% (370); trabajadores sociales, 6.0\% (258), y otras licenciaturas, 5.2\% (224). Usuarios internos, 46.1\% (1,969); externos, 41.0\% (1,751), y sin especificar procedencia, 12.9\% (553). Se encontró buena percepción sobre la calidad de los cursos en el $77.5 \%(3,310)$ de los usuarios; la evaluación de los docentes fue percibida como buena por el 100\% $(4,273)$ de los usuarios. Conclusión: No se encontró asociación entre edad y calidad percibida $\left(\chi^{2}: 164.05 ; p=0.06\right)$, pero sí entre perfil profesional y calidad percibida $\left(\chi^{2}: 72.78 ; p=0.00\right)$.

Palabras clave: Percepción. Calidad. Educación continua.

\section{Correspondence:}

*Patricia Rivera-Vázquez

E-mail: pattyri@live.com.mx
Date of reception: 30-10-2019

Date of acceptance: $28-01-2020$

DOI: 10.24875/HMCM.20000244
Available online: 02-04-2020 Hosp Med Clin Manag. 2020;13:13-9

2604-0018 / @ 2020 Regional Hospitals of High Specialty and Federal Hospitals. Published by Permanyer. This is an open access article under the CC BY-NC-ND license (http://creativecommons.org/licenses/by-nc-nd/4.0/). 


\section{INTRODUCCIÓN}

Uno de los compromisos fundamentales del personal de una institución de salud es la educación continua, que le permita contar con conocimientos actualizados para desarrollar su práctica con calidad y seguridad. La educación continua ha sido impulsada por la Organización Panamericana de la Salud desde 1986 en países de Latinoamérica ${ }^{1}$ como una estrategia de intervención pedagógica e institucional, orientada hacia la mejora de la organización y transformación de la práctica del equipo de salud, aprovechando los recursos disponibles humanos y tecnológicos para favorecer el proceso enseñanza-aprendizaje utilizando el potencial educativo que presenta problemas reales en situaciones laborales cotidianas.

La educación continua se define como un proceso continuo de actualización técnica y científica que ofrece al profesional la reflexión de la profesión, promueve el desarrollo personal y eleva la autoestima, además de facilitar la experimentación de la autonomía en el desempeño que acompaña al profesional como forma de estímulo y motivación. A la vez, puede posibilitar la mejora en la relación con el paciente y la familia, así como la comprensión de la enfermedad, debido al incentivo de la adquisición de conocimiento, despertando el autoconocimiento en el profesional2,3.

Su importancia es incluso mayor en la época actual debido a los cambios acelerados que experimentan los sistemas de salud. Ante esta situación, hay quienes consideran que es un derecho humano comparable con la libertad y el empleo, y que es deber del sistema de salud garantizarla a sus trabajadores para preservar la calidad de su desempeño y, de esta manera, garantizar a la sociedad ese otro derecho inalienable que es la salud ${ }^{4,5}$. Debe tomarse en cuenta que cálculos aproximados señalan que cada año aparecen alrededor de un $10 \%$ de conocimientos nuevos, un $50 \%$ es obsoleto en 5 años y el volumen del conocimiento médico útil se duplica cada 12 o 14 años y tiende a duplicarse en menos tiempo. Por otra parte, la práctica estereotipada de una disciplina y la curva del olvido contribuyen al deterioro de la capacidad del médico para su ejercicio diario, situaciones que no han pasado desapercibidas y hoy colocan a la educación médica continua como un proceso necesario para responder a los retos que la evolución y los avances que la ciencia y técnica imponen ${ }^{6}$.
La actualización profesional que hoy en día se realiza por medio de la educación continua surgió en el siglo XVII, cuando los primeros eruditos, especialmente clérigos, enseñaban a otros. En México, la Universidad Nacional Autónoma de México realizó en 1933 las primeras Jornadas Médicas para la educación médica continua en la Escuela Nacional de Medicina bajo la dirección del Dr. Ignacio Chávez; y en 1983 se creó la Comisión Interinstitucional para la Formación de Recursos Humanos para la Salud para desarrollar un sistema nacional de educación continua en las instituciones de salud en los diversos niveles de atención en nuestro país ${ }^{6}$. En 1998, la Asociación Nacional de Universidades e Instituciones de Educación Superior consideró a la educación continua como uno de los elementos útiles para el desarrollo de programas de seguimiento de egresados de las instituciones de educación superior que se encuentran insertados laboralmente en instituciones de servicio a la comunidad7,8.

Lo anterior establece que debemos conocer no solo los procesos de educación continua como un factor indispensable para asegurar la adecuación de los programas a las necesidades de cada institución de salud, tanto desde el punto de vista cuantitativo como cualitativo, sino también la percepción de los usuarios que permita a los educadores y planificadores proponer programas de educación continua que estipulen las diferentes actividades que deben organizarse para satisfacer las necesidades de los usuarios, con los recursos que sea factible movilizar dentro de una unidad de tiempo dada. La determinación de necesidades proporciona, además, la información básica para apreciar cambios en los niveles de salud, en la calidad y pertinencia del desempeño, y en el grado de satisfacción en el trabajo.

De acuerdo con la Organización Mundial de la Salud, una de las herramientas para alcanzar condiciones mínimas de prosperidad y bienestar está relacionada con la educación continua en salud. Por ello está presente en los objetivos para el desarrollo sostenible de la agenda 2015-2030 mediante garantizar una educación inclusiva y equitativa de calidad y promover oportunidades de aprendizaje permanente para todos en el contexto de las necesidades de recursos humanos para la salud, considerando como principios fundamentales la unidad de instrucción y educación, el concepto integral de la salud, la combinación de estudio y trabajo, así como la integración de la docencia, asistencia e investigación 
utilizando para ello métodos activos de enseñanzaaprendizaje. Además, se deben contemplar la dimensión biopsicosocial del hombre, el enfoque de acciones de promoción, prevención, curación y rehabilitación de la salud, y el pensamiento clinicoepidemiológico y ambientalista $^{9-11}$

En México, la Ley General de Salud recomienda a las autoridades educativas, en coordinación con las autoridades sanitarias en las instituciones de salud, participar de manera activa en la formación, capacitación y actualización del personal de salud, de conformidad con los objetivos y prioridades del Sistema Nacional de Salud, de los sistemas estatales de salud y de los programas educativos, en los diferentes niveles académicos y técnicos, mediante la promoción de un sistema de enseñanza continua en materia de salud ${ }^{12-15}$

Al respecto, autores mencionan que el deseo de aprender se observa en la mayor parte de los trabajadores de salud, ya que muchos de ellos son conscientes de su propia deficiencia y procuran remediarla; naturalmente, quienes no desarrollan esta consciencia constituyen el grupo de más alto riesgo de obsolescencia progresiva de la competencia profesional ${ }^{16}$. En este sentido, investigaciones realizadas mencionan que quien asiste con mayor frecuencia a los cursos de educación continua en salud es el personal femenino (en un $71 \%$ ), en comparación con el masculino (29\%). De los profesionales asistentes a los cursos la mayoría son enfermeras (82\%), seguido de médicos (11\%) y otros profesionales de salud (4\%). Solo una minoría de los asistentes posee algún posgrado $(20 \%)^{17-20}$. Con relación a la percepción que tiene el personal de salud sobre la calidad de los cursos de los programas de educación continua, algunos sectores mencionan que son de buena calidad, considerando que la actualización dentro de la profesión mediante la asistencia a diferentes eventos académicos es de gran importancia, ya que les permite estar al día en cuanto a conocimientos médicos, así como retroalimentarse en forma constante $20-22$

Mientras que en la evaluación general que realizan profesionales de salud de los cursos y programas de educación continua, reportan una asociación entre la percepción de calidad y el nivel académico del personal. Asimismo, con la edad, ya que a mayor edad mejor percepción de los cursos, no así con la categoría laboral y el nivel académico de los participantes ${ }^{21-26}$.
Por lo tanto, la percepción de los usuarios debe comprender un aprendizaje transformativo que implica producir agentes de cambio ilustrados, considerando que la calidad de los cursos ofrecidos mediante una educación efectiva construye cada nivel sobre el nivel previo. Como parte de un resultado con valor, el aprendizaje transformativo involucra tres desplazamientos fundamentales: de la memorización de hechos hacia la búsqueda, el análisis y la síntesis de la información que conduzca a la toma de decisiones; de la búsqueda de credenciales profesionales hacia la adquisición de competencias esenciales para un trabajo en equipo efectivo dentro de los sistemas de salud ${ }^{27,28}$; y de la adopción crítica de modelos educativos hacia una adaptación creativa de recursos globales para enfrentar prioridades locales.

Este es el caso del Hospital Regional de Alta Especialidad Bicentenario 2010 (HRAEV), en la región de Tamaulipas, que es una institución de referencia a nivel estatal y regional, donde de manera periódica asisten profesionales de la salud internos y externos a actualizar sus conocimientos, así como usuarios en formación que esperan nutrirse intelectualmente en cada uno de los cursos ofertados en el Programa de Educación Continua. Por tal motivo, el objetivo del presente estudio es determinar la asociación de la edad y perfil profesional de los asistentes con la percepción de la calidad de los cursos de educación continua en un hospital de tercer nivel de atención.

\section{MÉTODOS}

Se llevó a cabo una investigación no experimental de tipo cuantitativo realizada por medio de un estudio de tipo retrospectivo, observacional, transversal y descriptivo ${ }^{29}$ con usuarios asistentes a los cursos de educación continua del HRAEV durante el periodo de enero del 2013 a diciembre del 2017.

La población de estudio estuvo conformada por una muestra de 4,273 usuarios mediante muestreo no probabilístico, incluyendo profesionales y personal en formación en el área de salud de ambos sexos, que asistieron a los cursos de educación continua durante el periodo 2013-2017 y que contestaron el cuestionario denominado "Cédula de evaluación del evento educativo", el cual es un instrumento de elaboración institucional (HRAEV, 2013) que fue validado con un alfa de 
Tabla 1. Percepción de la calidad de los cursos de educación continua según la edad de los usuarios

\begin{tabular}{|c|c|c|c|c|c|}
\hline \multirow[t]{2}{*}{ Edad de los asistentes por grupo } & \multicolumn{4}{|c|}{ Evaluación del curso de educación continua } & \multirow[t]{2}{*}{ Total } \\
\hline & Bueno & Regular & Malo & No contestó & \\
\hline $18-24$ & 1,496 & 129 & 30 & 206 & 1,861 \\
\hline $25-31$ & 387 & 55 & 13 & 63 & 518 \\
\hline $32-38$ & 328 & 36 & 7 & 48 & 419 \\
\hline $39-45$ & 306 & 30 & 5 & 50 & 391 \\
\hline $46-52$ & 180 & 17 & 5 & 30 & 232 \\
\hline Más de 52 & 72 & 17 & 1 & 15 & 105 \\
\hline No especifica & 541 & 77 & 21 & 108 & 747 \\
\hline Total & 3,310 & 361 & 82 & 520 & 4,273 \\
\hline
\end{tabular}

Cronbach de 0.92, y que consta de tres apartados: a) datos personales, donde se registra sexo, edad, tipo de asistente (profesional o en formación), así como institución y zona de procedencia; b) evaluación del docente, en la que se califica por cada docente participante en el curso la puntualidad, explicación de los objetivos de la actividad, secuencia lógica de la exposición, dominio del tema, calidad de los materiales, manejo de las técnicas, participación y retroalimentación, dándose de 1 a 10 puntos, donde 1 es la menor calificación y 10 la máxima; y c) evaluación de la organización del evento, que valora la oportunidad de la realización del evento, horario, distribución del tiempo, puntualidad con la que se iniciaron las actividades, entrega de material de trabajo, funcionalidad de las instalaciones, organización general y apego a lo programado. Los resultados se consideraron como bueno (6-8 puntos), regular (3-5 puntos) o malo (0-2 puntos).

La recogida y procesamiento de los datos se realizaron mediante el programa Excel ${ }^{\circledR}$ (Microsoft, 2007) y el programa estadístico SPSS ${ }^{\circledR} 21.0$ (IBM) para la evaluación de la percepción de los usuarios sobre la calidad de los cursos de educación continua. El análisis de los datos de las variables sociodemográficas se realizó mediante estadística descriptiva con distribución de frecuencias y porcentajes. Para determinar la asociación entre las variables de estudio se utilizó estadística inferencial mediante tablas de contingencia, utilizando para ello la prueba de distribución también Ilamada chi cuadrada de Pearson $\left(\chi^{2}\right)$.

El desarrollo de la presente investigación estuvo apegado a lo dispuesto en la Declaración de Helsinki (2013). Asimismo, se apegó a las consideraciones éticas y legales enmarcadas en la Ley General de Salud en Materia de Investigación ${ }^{30}$ y se basó en las disposiciones de la Norma Oficial Mexicana 012 SSA3-2012, que establece los criterios para la ejecución de proyectos de investigación para la salud en seres humanos ${ }^{31}$.

\section{RESULTADOS}

Al término de la investigación, encontramos que durante el periodo 2013-2017 se desarrollaron 60 cursos de educación continua y 4,273 (82\%) usuarios respondieron la «Cédula de evaluación del evento educativo». Los asistentes fueron en su mayoría del sexo femenino $(3,277,76.7 \%)$, con una edad promedio de 24.37 años (rango: 19-60, desviación estándar: 14.489 años). La distribución de participantes mostró predominio de enfermeras $(1,086,25.4 \%)$ y pasantes de enfermería $(1,014,23.7 \%)$, seguidos de estudiantes universitarios (598, 14\%), médicos (370,8.7\%), trabajadores sociales $(258,6 \%)$, de otras licenciaturas $(224,5.2 \%)$ y sin especificar $(723,16.9 \%)$. Los usuarios fueron 1,969 internos (46.1\%), 1,751 externos al HRAEV (41\%) y 553 sin especificar institución de procedencia (12.9\%) (Tabla 1).

Con relación a las variables de estudio, encontramos que 3,590 (84\%) usuarios percibieron que la oportunidad de la realización del evento fue buena, para 138 $(3.2 \%)$ fue regular, para $12(0.3 \%)$ fue mala y 533 (12.5\%) no contestaron la pregunta.

Respecto al horario de los cursos, 3,313 (77.5\%) lo percibieron como bueno, 397 (9.3\%) regular y 38 (0.9\%) malo; 525 usuarios (12.3\%) no respondieron.

En cuanto a la distribución del tiempo en que se desarrollaron los cursos de educación continua, 3,244 (75.9\%) mencionaron que fue bueno, 453 (10.6\%) regular, 53 $(1.2 \%)$ malo y $523(12.2 \%)$ no contestaron la pregunta. 
Tabla 2. Percepción de la calidad de los cursos de educación continua según el perfil profesional de los asistentes

\begin{tabular}{|c|c|c|c|c|c|}
\hline \multirow[t]{2}{*}{ Perfil profesional } & \multicolumn{4}{|c|}{ Evaluación del curso de educación continua } & \multirow[t]{2}{*}{ Total } \\
\hline & Bueno & Regular & Malo & No contestó & \\
\hline Médico(a) & 253 & 45 & 8 & 64 & 370 \\
\hline Enfermera(0) & 859 & 79 & 20 & 128 & 1,086 \\
\hline Trabajador(a) social & 207 & 16 & 7 & 28 & 258 \\
\hline Universitarios(as) & 505 & 21 & 6 & 66 & 598 \\
\hline Pasantes & 780 & 87 & 21 & 126 & 1,014 \\
\hline Otras licenciaturas & 183 & 23 & 3 & 15 & 224 \\
\hline No especifica & 523 & 90 & 17 & 93 & 723 \\
\hline Total & 3,310 & 361 & 82 & 520 & 4,273 \\
\hline
\end{tabular}

Sobre la puntualidad en que dieron inicio las actividades de los cursos, 3,289 (77\%) mencionaron que fue buena, $416(9.7 \%)$ regular, $46(1.1 \%)$ mala y $522(12.2 \%)$ no contestaron la pregunta. Respecto a la entrega de material de los cursos, 3,463 (81\%) dijeron que fue bueno, $236(5.5 \%)$ regular, 50 (1.2\%) malo y no contestaron la pregunta 524 (12.3\%) usuarios. La funcionalidad de las instalaciones fue percibida como buena por 3,531 (82.6\%) usuarios, 188 (4.4\%) como regular, 32 (0.7\%) mala y no contestaron la pregunta $522(12.2 \%)$ de los usuarios (Tabla 2).

La organización del evento académico fue percibida como buena por 3,426 (80\%) usuarios, regular por 282 (6.6\%) y mala por $46(1.1 \%)$; no contestaron la pregunta $519(12.1 \%)$ usuarios. El apego a la programación fue percibido como bueno por 3,250 (76.1\%), regular por $434(10.2 \%)$ y malo por 68 (1.6\%); 521 (12.2\%) usuarios no contestaron la pregunta (Fig. 1).

La percepción general de la calidad de los cursos del programa de educación continua fue percibida como buena por 3,310 (77.5\%) usuarios, regular por 361 (8.4\%) y mala por 82 (1.9\%); no contestaron 520 (12.2\%) usuarios. Respecto a la evaluación de los docentes, 4,273 (100\%) usuarios los evaluaron como buenos considerando los diferentes indicadores para cada uno de los ponentes participantes en los cursos, siendo el año 2015 el mejor evaluado por los usuarios (Fig. 2).

Al analizar los datos para determinar la asociación entre la edad de los asistentes y la calidad percibida encontramos que no existe asociación entre las variables $\left(\chi^{2}: 164.05 ; p=0.06\right.$ ) (Tabla 1 ). Con relación al perfil profesional, encontramos que sí existió asociación entre perfil profesional y calidad percibida $\left(\chi^{2}: 72.78\right.$; $p=0.00$ ) (Tabla 2).

\section{Percepción acerca del apego a la programación de los cursos}

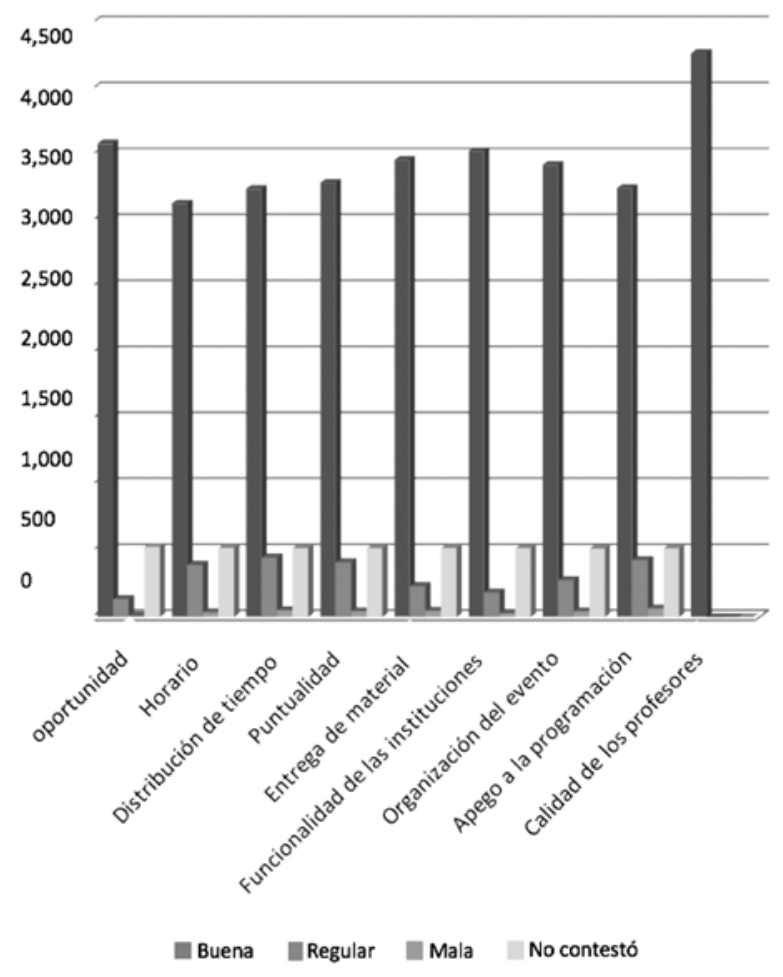

Figura 1. Percepción acerca del apego a la programación de los cursos.

\section{DISCUSIÓN}

La mayoría (82\%) de los usuarios asistentes a los cursos de educación continua en el HRAEV contestó la cédula de evaluación, lo que nos permitió identificar la percepción sobre la calidad de los cursos realizados. Esto fue similar a lo descrito en la literatura, considerando que la actualización por medio de diferentes eventos académicos permite estar al día en cuanto a conocimientos 


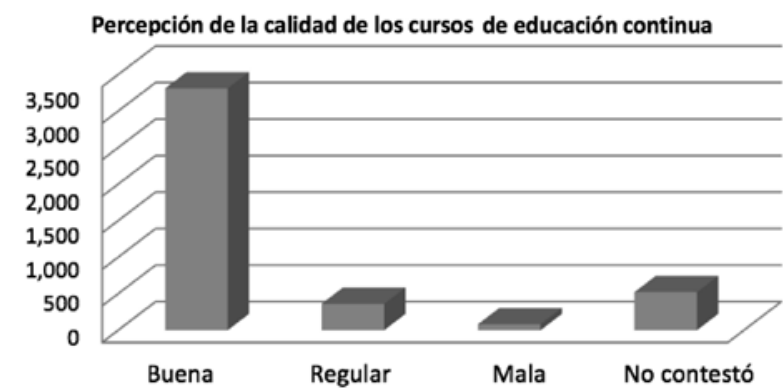

Figura 2. Percepción de la calidad de los cursos de educación continua.

relacionados a la salud y la profesión de cada uno, así como retroalimentarse en forma constante ${ }^{20}$.

En cuanto a los usuarios, la mayoría fueron del sexo femenino $(3,277,76.7 \%)$, similar a lo descrito en otros estudios $^{17-19}$. En la investigación encontramos que los usuarios que asisten a nuestros cursos tienen una edad promedio de 24.3 años, diferente a lo encontrado por algunos autores ${ }^{17,21}$, quienes mencionan que los asistentes a los cursos de educación continua en salud generalmente son adultos mayores de 45 años.

Con relación al perfil profesional, encontramos predominio de usuarias enfermeras $(25.4 \%)$ y pasantes de enfermería (23.7\%), similar a lo descrito en la literatura $^{18-20}$, donde se hace mención que es el personal de enfermería quien mayormente asiste a cursos de educación continua, especialmente al inicio de su desarrollo profesional, en las instituciones de salud que los ofertan, siendo esto lo observado, ya que el $46.1 \%$ fueron usuarios del propio HRAEV.

La calidad de los cursos del programa de educación continua fue percibida como buena por el $77.5 \%$ de los usuarios, similar a lo reportado en la literatura ${ }^{20,21}$. Respecto a la evaluación de los docentes, el 100\% de los usuarios percibió una buena calidad de los profesores participantes. Al respecto, no encontramos literatura específica con la cual discutir los resultados obtenidos.

Al analizar la percepción de la calidad de los cursos de educación continua, encontramos que no existe asociación entre edad y calidad percibida $\left(\chi^{2}: 164.05 ; p=0.06\right)$, contrario a lo encontrado por otros autores ${ }^{21-26}$, quienes además estudiaron otras variables no revisadas en este estudio como son el puesto laboral y los años de servicio, pero sin encontrar asociación al respecto. Con relación al perfil profesional, encontramos que sí existe asociación entre tipo de profesional y calidad percibida $\left(\chi^{2}: 72.78 ; p=0.00\right)$, similar a lo encontrado en la literatura ${ }^{21,24}$, en donde se ha reportado asociación con el perfil profesional de los trabajadores de la salud.

\section{CONCLUSIÓN}

Los asistentes al HRAEV percibieron una buena calidad de los cursos de educación continua. Se caracterizan por ser en su mayoría del sexo femenino (76.7\%), con una edad promedio de 24.37 años y de procedencia interna (46.1\%), con perfil profesional de enfermería y prestatarios de servicio social. No se encontró asociación entre edad y calidad percibida ( $\chi^{2}:$ 164.05; $p=0.06$ ), pero sí entre tipo de profesional y calidad percibida $\left(\chi^{2}: 72.78 ; p=0.00\right)$.

La investigación realizada permitió no solo conocer la percepción de los usuarios como una oportunidad de mejora de la calidad de los cursos, sino dar lugar a nuevas estrategias que detecten sugerencias por parte de los usuarios, mediante buzón de opinión u otro medio, sistematizando la información por medio de base de datos y promoviendo nuevos estudios de investigación relacionados con la actividad académica del hospital. Con ello se busca hacer más eficientes las actividades derivadas de los convenios de colaboración para realizar investigación en red con otras instituciones de salud y educativas del país.

Trabajo de investigación presentado en modalidad de cartel en el 1. ${ }^{\text {er }}$ Congreso Internacional y VIII Congreso Nacional de Educación Médica, durante los días 24, 25 y 26 de octubre de 2018 en el Instituto Nacional de Enfermedades Respiratorias Ismael Cosío Villegas, en Ciudad de México.

\section{CONTRIBUCIÓN}

SHDE: introducción, objetivos de investigación y revisión documental.

RVP: diseño metodológico, captura y análisis estadístico, elaboración de discusión y conclusión.

RGS: asesoría externa. 


\section{AGRADECIMIENTOS}

A las autoridades del HRAEV por las facilidades otorgadas para la realización del estudio.

\section{FINANCIAMIENTO}

La presente investigación no ha recibido beca específica de agencias de los sectores público, comercial o sin ánimo de lucro.

\section{CONFLICTO DE INTERESES}

Ninguno.

\section{RESPONSABILIDADES ÉTICAS}

Protección de personas y animales. Los autores declaran que para esta investigación no se han realizado experimentos en seres humanos ni en animales.

Confidencialidad de los datos. Los autores declaran que en este artículo no aparecen datos de pacientes.

\section{Derecho a la privacidad y consentimiento informado.} Los autores declaran que en este artículo no aparecen datos de pacientes.

\section{BIBLIOGRAFÍA}

1. Mejía A. Educación continua. Educ Med Salud. 1986;20(1):43-71.

2. Sardinha Peixoto L, Cuzatis Gonçalves L, Dutra Da Costa T, Tavares CMDM, Dantas Cavalcanti AC, Antunes Cortez E. Educación permanente, continuada y de servicio: desvelando sus conceptos. Enfermería Glob. 2013;12(29): 307-22.

3. Barbón Pérez 0, Fernández Pino JW. Rol de la gestión educativa estratégica en la gestión del conocimiento, la ciencia, la tecnología y la innovación en la educación superior. Rev Educ Med. 2018:19(1):51-5.

4. Roschke MA. Evaluación en procesos de educación permanente y capacitación en salud. Experiencias y lecciones. Washington DC: Organización Panamericana de la Salud-Organización Mundial de la Salud; 2006.

5. Nsemo AD, John ME, Etifit RE, Mgbekem MA, Oyira EJ. Clinical nurses' perception of continuing professional education as a tool for quality service delivery in public hospitals, Calabar, Cross River State, Nigeria. Nurse Educ Pract. 2013;13(4):328-34.

6. Saturno-Hernández PJ, Hernández-Ávila M, Magaña-Valladares L, GarcíaSaisó S, Vértiz-Ramírez JJ. Estrategia integral de formación para la mejora continua de la calidad de los servicios de salud. Salud Pub Mex. 2015;57:275-83
7. Landgrave Ibáñeza MR, Trabanino Mata FJ, Lizcano Esperón EJ, Yáñez Puiga R, Méndez U, Ponce Rosas ER. La formación médica continuada en México. Aten Primaria. 2002;29(3):178-88.

8. Velasco MN. La educación médica continua como herramienta constante de aprendizaje. Rev Chil Cir. 2013;65(2):187-91.

9. Ricaldoni CAC, Sena Roseni R. Educación permanente: una herramienta para pensar y actuar en el trabajo de enfermería. Rev Latino-Am Enfermagem. 2006;14(6):837-42.

10. Bello Rodríguez B, Báez Pérez EG, Sánchez Cruz G, Fernández Morín J, Fernández Alonso A, Delgado Pérez L. La concepción integral de la salud: principio de la educación en ciencias de la salud. Rev Med Electon. 2011;33(6):807-16

11. Mikael SSE, Cassiani SHDB, Silva FAM. The PAHO/WHO Regional Network of Interprofessional Health Education. Rev Latino-Am Enfermagem. 2017;25:2866.

12. Organización de las Naciones Unidas para la Educación, la Ciencia y la Cultura. Fortaleciendo competencias: Formación continua para el Programa Abriendo Espacios. Educación y Cultura para la Paz. Organización de las Naciones Unidas para la Educación, la Ciencia y la Cultura; 2009.

13. Ley General de Salud. Nueva Ley publicada en el Diario Oficial de la Federación el 7 de febrero de 1984. Texto vigente. Últimas reformas publicadas DOF 24 01-2020 [Internet]. Estados Unidos Mexicanos, Cámara de Diputados del H. Congreso de la Unión, Secretaría General [fecha de publicación: 24/01/2020]. Disponible en: http://www.diputados.gob.mx/LeyesBiblio/pdf/142 240120.pdf

14. Pastor Arango ME. La educación permanente en la seguridad y salud en el trabajo. Rev Cub de Salud y Trabajo. 2017:18(2):57-9.

15. Mejía A. Importancia de la educación continua. Acta Med Col. 1985;10(2).

16. Karchmer KS. Educación médica continuada. Necesidades de proyección. Acta Med. 2006;4(1).

17. Treviso P, Pinheiro da Costa BE. Percepção de profissionais da área da saúde sobre a formação em sua atividade docente. Rev Texto Contexto Enferm. 2017;26(1).

18. Fernández Pérez J. Educación médica continua y desarrollo de una profesión. La percepción de los actores. Rev Ed Desarr. 2014;28(2):21-35.

19. Calvo-Calvo M. Características de la formación continuada impartida a profesionales de la salud. Rev FEM. 2013;16(3):137-44.

20. Muñoz Casto FA, Valverde-Gambero L, Villanueva-Guerrero MJ, MudarraAceituno M, Vázquez-Vázquez, Almuedo PA. Evolución de la formación continuada acreditada tras la puesta en marcha de la Estrategia para la Seguridad del Paciente. Rev Calid Asist. 2012;27(5):262-9.

21. Aliyu A, Ibrahim TA, Danjuma A, Tawheed M. Perspectives of continuing formal education among nurses in selected secondary healthcare facilities in Northern Nigeria. Am J Health Res. 2015;3(1-1):68-73.

22. Cadena Estrada C, Olvera Arreola SS, Pérez López MT, Balseiro Almario C, Matus Miranda R. Evaluación de la capacitación de enfermería en dos institutos nacionales de salud. Enfermería Universitaria. 2012:9(3):15-24.

23. González Guzmán R. Nueve puntos para la reflexión educativa. Rev Inv Ed Med. 2013;2(1):42-9.

24. Karchmer S. Educación médica continuada: necesidades de proyección. Acta Med. 2006;4(1).

25. Hamui-Suttona A. Varela-Ruiz M, Ortiz-Montalvo A, Torruco-García E. Las actividades profesionales confiables: un paso más en el paradigma de las competencias en educación médica. Rev Fac Med UNAM. 2015;58(5):25-39.

26. Feijò de Andrade IC. Educación permanente y continuada en una perspectiva de integridad del ser. Rev Edu. 2014;9(2):142-50.

27. Frenk J, Chen L, Zulfigar, Bhuta A, Cohen J, Crisp N. Profesionales de la salud para el nuevo siglo: Transformando la educación para fortalecer los sistemas de salud en un mundo independiente. Educ Med. 2015;16(1):9-16.

28. Vélez Benito GA, Schwabe Franz M. Educación permanente en salud. Reflexiones en la perspectiva de la integridad. Rev Cub Enfermer. 2010;26(4):667-79.

29. Hernández Sampieri R. Metodología de la Investigación. 6. ${ }^{a}$ ed. Mc GrawHill; 2014. pp. 126-168.

30. Reglamento de la Ley General de Salud en Materia de Investigación para la Salud. Nuevo Reglamento publicado en el Diario Oficial de la Federación el 6 de enero de 1987. Texto vigente. Última reforma publicada DOF 02-04-2014 [Internet]. Estados Unidos Mexicanos, Cámara de Diputados del H. Congreso de la Unión, Secretaría General [fecha de publicación: 02/04/2014]. Disponible en: http://www.diputados.gob.mx/LeyesBiblio/regley/Reg_LGS_MIS.pdf

31. Norma Oficial Mexicana NOM-012-SSA3-2012, Que establece los criterios para la ejecución de proyectos de investigación en seres humanos [Internet]. Estados Unidos Mexicanos: Secretaría de Gobernación, Secretaría de Salud, Diario Oficial de la Federación [fecha de publicación: 04/01/2013]. Disponible en: https://dof.gob.mx/nota_detalle.php?codigo=5284148\&fecha=04/01/2013 